16 a 18 de outubro de 2019 - Campinas | Brasil

\title{
Comparison between experimental and simulated phase envelopes of the methane/olefin mixture
}

\section{André T. P Falcade*, Nara A. Policarpo, Nilo R. Kim, Paulo R. Ribeiro}

\section{Abstract}

The study aims to compare bubble pressures of methane/olefin mixture obtained experimentally with those simulated by WinProp $\AA$, building the respective phase envelopes for different methane molar fractions, pressures, and temperatures.

\section{Key words:}

Olefin, Phase envelopes, WinProp®.

\section{Introduction}

In well control, quantifying the formation gas dissolved in the drilling fluid is essential to avoid kicks. In the case of deep wells, where pressures and temperatures are high, it becomes even more fundamental to know PVT (pressure-volume-temperature) behavior of the gas/drilling fluid mixture, since the gas solubility can be infinity and its quantification more difficult at the supercritical regions ${ }^{1}$. For mixtures, the PVT behavior can be studied from its phase envelopes, which are regions that make up the phase diagram of a mixture, where the liquid and gas phases coexist, delimited by the bubble and dew lines. At the critical point, there is no liquid/gas interface and, above critical pressure and critical temperature, only one phase exists, called the supercritical region. In it, the fluid acquires intermediate properties of liquids and gases ${ }^{2}$. Phase envelopes can be experimentally obtained or through compositional phase behavior simulators, such as the PVT Pro (Schlumberger) and the CMG WinProp $\AA$. This work aims to compare the bubble pressures of the methane (formation gas) and olefin (drilling fluid base) mixtures obtained experimentally with those simulated by WinProp®.

\section{Results and Discussion}

Experimental saturation pressures $\left(\mathrm{P}_{\text {sat }}\right)$ or bubble points were measured in a PVT cell for the methane/olefin mixtures. The results were obtained for methane molar fraction of 10 to $40 \%$ and temperatures of $20,25,40,60$ and $80^{\circ} \mathrm{C}$, that resemble petroleum reservoir conditions. The PVT results were feed into the CMG WinProp® (version 2016.1) simulator to obtain the phase envelopes of the methane/olefin mixture. The Equation of State (EOS) used was the Peng-Robinson and the binary interaction parameter $\left(\mathrm{k}_{\mathrm{ij}}\right)$ is calculated by correlation ${ }^{3}$ shown in Equation 1, where $v_{c}$ is the critical volume of species $\mathrm{i}$ or $\mathrm{j}$ and $\theta$ is a parameter adjustable for the mixture.

$$
\mathrm{k}_{\mathrm{ij}}=1-\frac{\left(2 \mathrm{v}_{\mathrm{ci}}^{1 / 6} \mathrm{v}_{\mathrm{cj}}^{1 / 6}\right)^{\theta}}{\left(\mathrm{v}_{\mathrm{ci}}^{1 / 3}+\mathrm{v}_{\mathrm{cj}}^{1 / 3}\right)}
$$

The simulated phase envelopes were obtained for two situations: (i) adjustment of the EOS through regression with the PVT experimental values, and (ii) without regression. Image 1 compares the phase envelopes obtained for the two mentioned situations and shows the saturation pressures experimentally measured. The figure shows that simulated bubble pressures are more accurate when the EOS is tuned by experimental data.

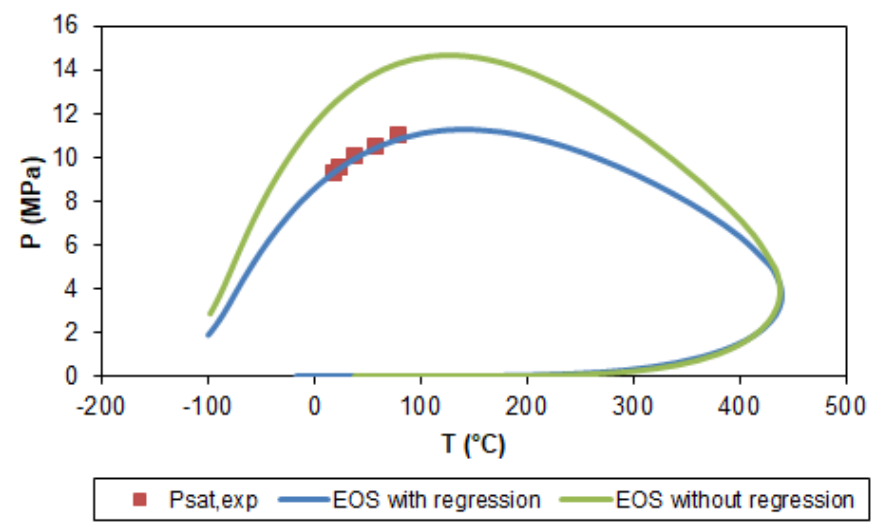

Image 1. Phase envelope of methane/olefin mixture - 10\% of methane.

Chart 1 presents $P_{\text {sat }}$ obtained experimentally and simulated. All deviation values between experimental and simulated $P_{\text {sat }}$ (bubble points) were lower than $10 \%$, except for the methane molar fraction of $30 \%$ that was $11 \%$ of error.

Chart 1. Experimental and simulated bubble points (MPa).

\begin{tabular}{|l|c|c|c|c|c|c|c|c|}
\hline \multirow{2}{*}{$\mathbf{T}\left({ }^{\circ} \mathbf{C}\right)$} & \multicolumn{8}{|c|}{ Methane Molar Fraction (\%) } \\
\cline { 2 - 9 } & \multicolumn{2}{|c|}{$\mathbf{1 0}$} & \multicolumn{2}{|c|}{$\mathbf{2 0}$} & \multicolumn{2}{|c|}{$\mathbf{3 0}$} & \multicolumn{2}{|c|}{$\mathbf{4 0}$} \\
\cline { 2 - 9 } & Exp & Sim & Exp & Sim & Exp & Sim & Exp & Sim \\
\hline 20 & 9,2 & 9,4 & 23,8 & 23,6 & 24,0 & 26,7 & 36,1 & 39,6 \\
\hline 25 & 9,5 & 9,6 & 24,0 & 23,7 & 24,3 & 26,6 & 36,1 & 38,8 \\
\hline 40 & 10,0 & 10,0 & 24,5 & 23,9 & 24,8 & 26,3 & 36,5 & 37,0 \\
\hline 60 & 10,4 & 10,5 & 25,3 & 24,1 & 25,5 & 26,2 & 36,1 & 35,5 \\
\hline 80 & 11,0 & 10,9 & 25,7 & 24,1 & 26,2 & 26,2 & 35,5 & 34,5 \\
\hline
\end{tabular}

\section{Conclusions}

It is concluded that the phase envelopes generated from experimental data better represent the PVT behavior of the methane/olefin mixture, under the conditions described in this work.

\section{Acknowledgement}

The authors are thankful for Petrobras financial support. 